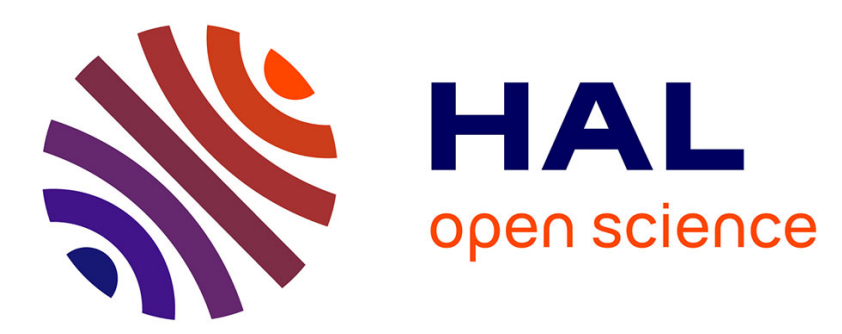

\title{
Aspects physico-chimiques de la préparation de couches minces semiconductrices par condensation de plasmas froids
}

\author{
B. Laville Saint Martin, G. Perny, M. Samirant, P. van den Berghe
}

\section{- To cite this version:}

B. Laville Saint Martin, G. Perny, M. Samirant, P. van den Berghe. Aspects physico-chimiques de la préparation de couches minces semiconductrices par condensation de plasmas froids. Revue de Physique Appliquée, 1966, 1 (3), pp.230-236. 10.1051/rphysap:0196600103023001 . jpa-00242723

HAL Id: jpa-00242723

https://hal.science/jpa-00242723

Submitted on 1 Jan 1966

HAL is a multi-disciplinary open access archive for the deposit and dissemination of scientific research documents, whether they are published or not. The documents may come from teaching and research institutions in France or abroad, or from public or private research centers.
L'archive ouverte pluridisciplinaire HAL, est destinée au dépôt et à la diffusion de documents scientifiques de niveau recherche, publiés ou non, émanant des établissements d'enseignement et de recherche français ou étrangers, des laboratoires publics ou privés. 


\title{
ASPECTS PHYSICO-CHIMIQUES \\ DE LA PRÉPARATION DE COUCHES MINCES SEMICONDUCTRICES PAR CONDENSATION DE PLASMAS FROIDS
}

\author{
Par B. LAVILLE SAINT MARTIN, G. PERNY, M. SAMIRANT \\ et P. VAN DEN BERGHE, \\ Laboratoire de Physico-Chimie des Couches Minces Solides, Mulhouse.
}

\begin{abstract}
Résumé. - Étude de l'influence des variations du champ réduit $E^{\star}$ et de la concentration en oxygène des plasmas froids $(\mathrm{Cu}, \mathrm{A}, \mathrm{O})^{\star}$ et $(\mathrm{Ti}, \mathrm{A}, \mathrm{O})^{\star}$ sur les caractéristiques des couches minces condensées sur des supports solides ; examen de leurs propriétés par différentes techniques et mise en évidence $d^{\prime}$ une épaisseur caractéristique du couple support-couche mince. Proposition d'une théorie phénoménologique de la condensation des plasmas froids, basée sur l'hypothèse de l'existence de réactions chimiques collisionnelles et comparaison avec les résultats expérimentaux dans le cas $(\mathrm{Ti}, \mathrm{A}, \mathrm{O})^{\star}$.
\end{abstract}

Abstract. - Study of the influence of the reduced field $E^{\star}$ and of the oxygen ratio of cold plasmas $(\mathrm{Cu}, \mathrm{A}, \mathrm{O})^{\star}$ and $(\mathrm{Ti}, \mathrm{A}, \mathrm{O})^{\star}$ upon the characteristics of thin films condensed on solid substrates ; investigation of their properties by different methods. Evidence for the existence of a thickness specific to the substrate-film couple. Proposition of a phenomenological theory of the condensation of cold plasmas based on the hypothesis of collisional chemical reactions and comparison with experimental results in the case of $(\mathrm{Ti}, \mathrm{A}, \mathrm{O})^{\star}$.

Des couches minces métalliques, semiconductrices, supraconductrices ou diélectriques peuvent être préparées de différentes manières, groupées selon les auteurs en méthodes chimiques, thermiques ou électriques. Pour des substances binaires on a utilisé avec avantage les principes de l'évaporation réactive (E. R.) [1]. Dans le cas particulier où l'utilisation d'une décharge électrique intervient pour initier les dépôts de synthèse, on parle de pulvérisation cathodique réactive (P.C. R.) [2].

Il nous a semblé dès lors que la multiplicité des techniques invoquées pouvait, par la conjonction des notions de synthèse en phase gazeuse et de P. C. R., donner lieu à une définition plus générale et par conséquent génératrice de recherches nou- velles, c'est la notion de "Condensation de Plasmas Froids ". Elle se place dans le cadre d'une PhysicoChimie des Couches Minces, discipline issue des nécessités de la technologie moderne, dont l'une est la reproductibilité des résultats.

C'est dans cet ordre d'idées que nous proposons d'évoquer certains aspects d'ordre physico-chimique liés à la préparation de couches minces semiconductrices de $\mathrm{Cu}_{2} \mathrm{O}$ et $\mathrm{TiO}_{2}$.

L'obtention systématique de couches minces à caractéristiques reproductibles à partir de la condensation de plasmas froids (métal, argon, oxygène) ${ }^{*}$, est conditionnée par la mise en cuvre d'études partjculières et approfondies consacrées à la cathode, au 
plasma, au support et à la couche condensée. Cette condensation, iniliée sous pression réduite, aboutit, selon les conditions expérimentales et à la température ambiante, au dépôt de couches minces du métal ou de l'un ou l'autre de ses oxydes.

La nature chimique du condensat dépend de nombreux paramètres et singulièrement du pourcentage $C$ d'oxygène contenu dans le mélange gazeux réactionnel et de la valeur du champ réduit $E^{*}=\frac{E}{P} \cdot\left(\mathrm{Vm}^{-1} \mathrm{~Pa}^{-1}\right)$.

La mise en corrélation de ces deux grandeurs avec le rendement $\bar{\eta}$ de la condensation permet de tracer un diagramme d'équilibre tridimensionnel $\bar{\eta}=f\left(C, E^{*}\right)$ (fig. 1) [3] qui révèle l'existence de

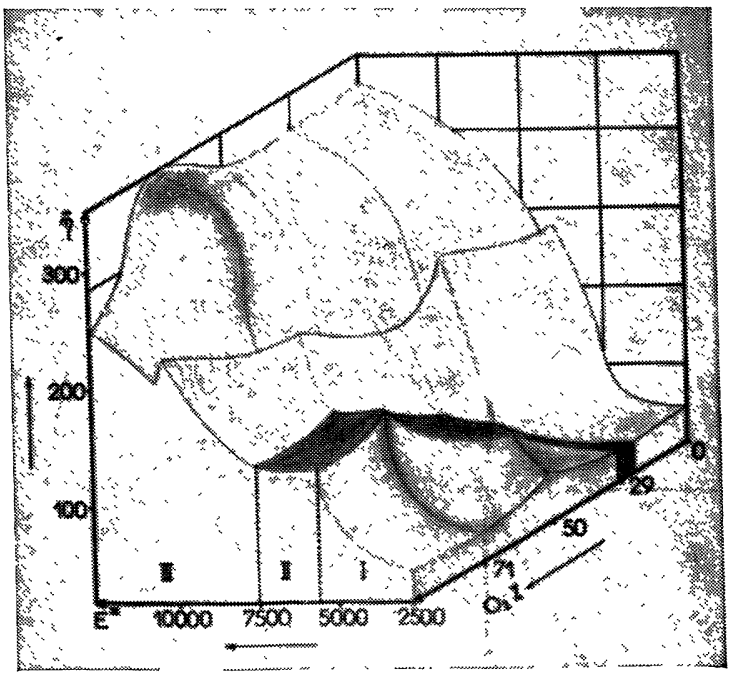

Fig. 1. - Diagramme d'équilibre

de la condensation du plasma froid $(\mathrm{Cu}, \mathrm{A}, \mathrm{O})^{*}$.

domaines privilégiés correspondant chacun à la formation préférentielle de couches minces du métal ou de ses oxydes. Un exemple est fourni par la condensation du plasma $(\mathrm{Cu}, \mathrm{A}, \mathrm{O})^{*}$ où les domaines, correspondent respectivement à la synthèse de $\mathrm{Cu}_{2} \mathrm{O}$ (I), $\mathrm{CuO}$ (II) et au dépôt de $\mathrm{Cu}$ pur (III). Le diagramme $E^{*}=f(C)$ se présente alors comme un diagramme de phase (fig. 2) où la grandeur énergétique classique de la chimie est remplacée par la grandeur $E^{*}[3]$.

Des mesures spécifiques, comme celles de résistivité électrique, permettent de mettre en évidenc6 le degré de reproductibilité des propriétés de ces couches, ainsi que leur taux de stoechiométrie (fig. 3).

Une propriété remarquable réside dans le fait que les couches obtenues par cette technique sont beaucoup plus adhérentes que celles obtenues par évaporation thermique. Cette circonstance trouve son explication dans les grandes énergies des projectiles

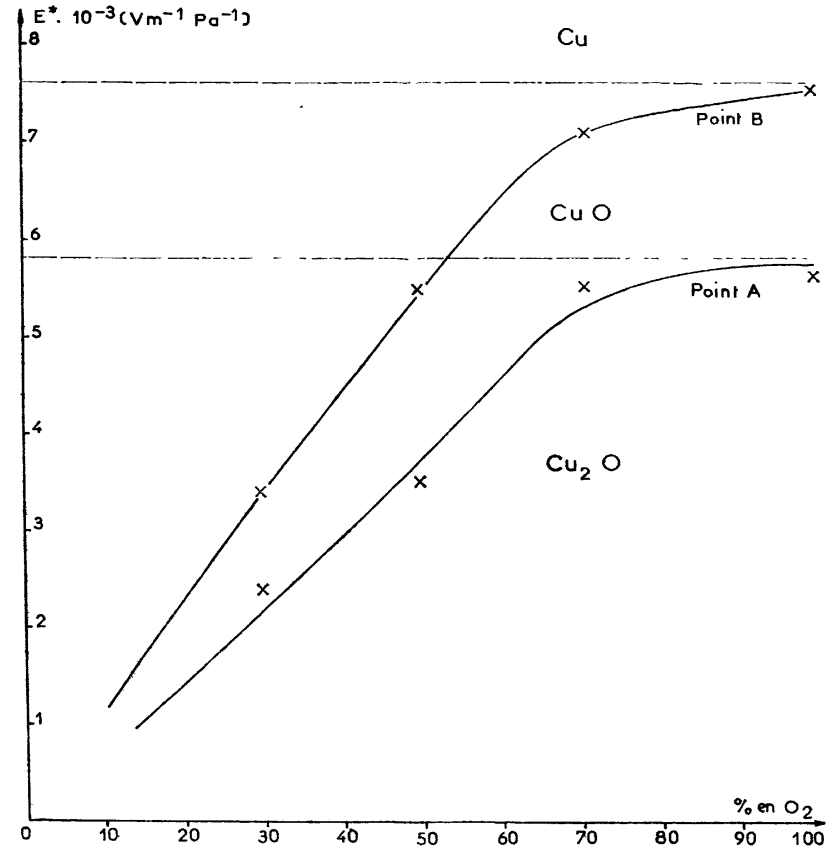

Fig. 2. - Diagramme de phases. Variation des champ réduits caractéristiques avec la concentration en $\mathrm{O}_{2}$

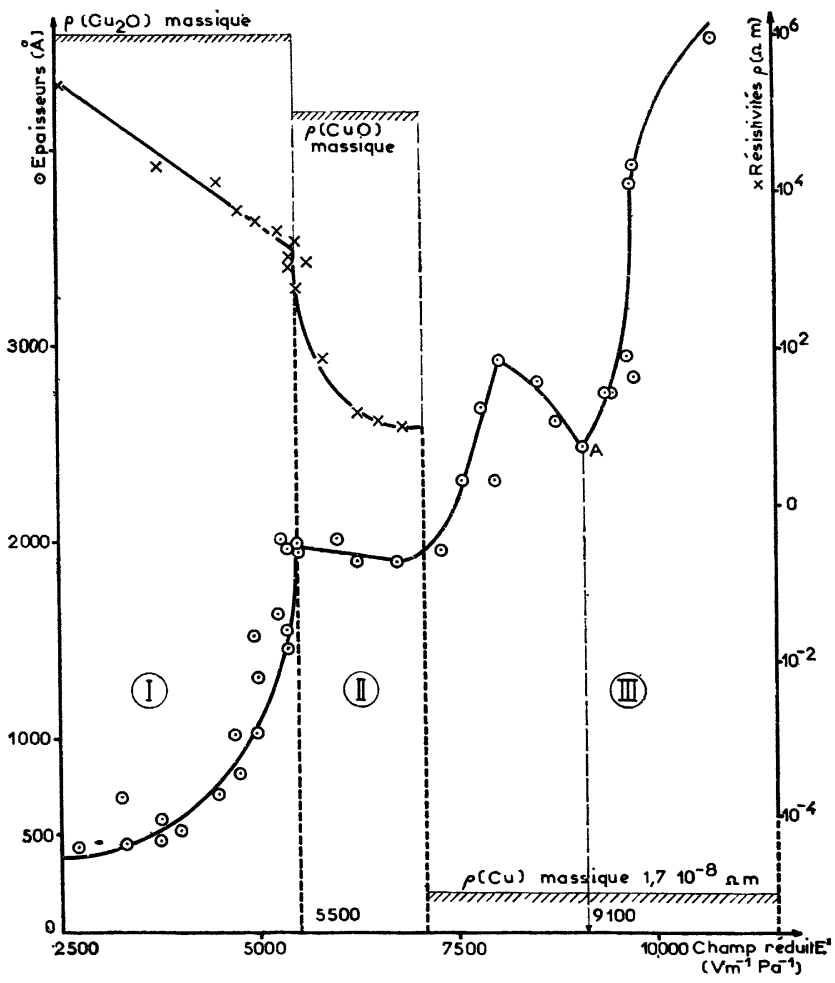

Fig. 3. - Synthèse de couches minces d'oxydes de cuivre. Concentration : $71 \% \mathrm{O}_{2}, 29 \%$ A. Temps $15 \mathrm{mn}$. Cathode cuivre, support $\mathrm{Si} \mathrm{O}_{2}$. 
du plasma. Celles-ci favorisent en effet l'apparition de sites de germination sur les supports, comme nous l'avons montré dans le cas de la silice amorphe [4].

Les oxygènes radicalaires créés au niveau des sites superficiels de la silice sous l'action du bombardement ionique, participent au mécanisme d'adhérence des couches d'oxydes de métaux. Il se forme, dans tous les cas, une zone de transition entre le support et la matière condensée, zone dont l'épaisseur dépend de la nature du couple substratcondensat [3] et dont les propriétés physico-chimiques sont différentes de celles de la couche mince proprement dite comme nous le verrons lors de l'étude des dépôts par fluorescence de rayons $\mathrm{X}$.

Pour tenter de mettre en évidence les phénomènes provenant de l'existence de couches de transition et par conséquent ceux de la germination, nous avons examiné par diffraction $\mathrm{X}$ des couches minces de $\mathrm{Cu}$ préparées par pulvérisation cathodique [5]. Nous avons observé que les variations de la taille des germes, calculée par la formule de Scherrer, n'évoluaient pas d'une façon monotone en fonction des épaisseurs des couches. On observe en effet qu'à une épaisseur de l'ordre de $900 \AA$ dans le cas de $\mathrm{Cu}$ projeté dans $100 \%$ A et de $1000 \AA$ dans le cas de $\mathrm{Cu}$ projeté dans $100 \% \mathrm{O}_{2}$, la taille des cristallites passe par un extrêmum de $140 \AA$ et $160 \AA$ respectivement. D'autre part les mesures d'intensités des plans (111) et (002) du cuivre montrent qu'il existe des croissances privilégiées même sur support amorphe [5], [6].

Nous avons également étudié des couches minces d'oxydes de titane non stoechiométriques et de Ti métallique par fluorescence de rayons $X$ [7]. Nous avons établi la corrélation entre des mesures optiques

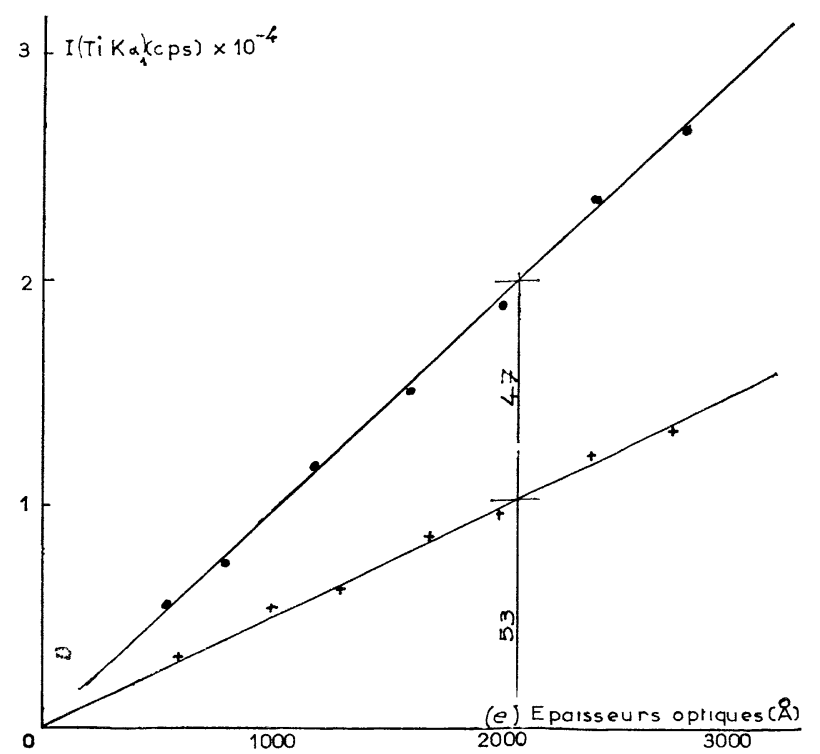

Fig. 4. $-\Delta I\left(\mathrm{Ti} K \alpha_{1}\right)=f(\Delta e)+, \mathrm{TiO}_{2}$ sur $\mathrm{Si} \mathrm{O}_{2}$. -, Ti sur $\mathrm{SiO}_{2}$ conditions : $30 \mathrm{kV}, 20 \mathrm{~mA}$. d'épaisseurs et celles qui sont basées sur l'émission $\mathrm{TiK} \alpha_{1}$ des couches. Les écarts de corrélation observés ont pu être attribués à deux phénomènes distincts se rapportant d'une part à la composition chimique (fig. 4) et d'autre part à la texture des couches (fig. 5-6). On définit à ce propos un coefficient de transformation $b$ tel que

$$
b=\frac{I}{I_{0}} \frac{\mathrm{d} I_{\mathrm{m}}}{\mathrm{d} e}
$$

$I_{0}=$ intensité du faisceau incident,

$I_{\mathrm{m}}=$ intensité de fluorescence mesurée,

$e=$ épaisseur optique.

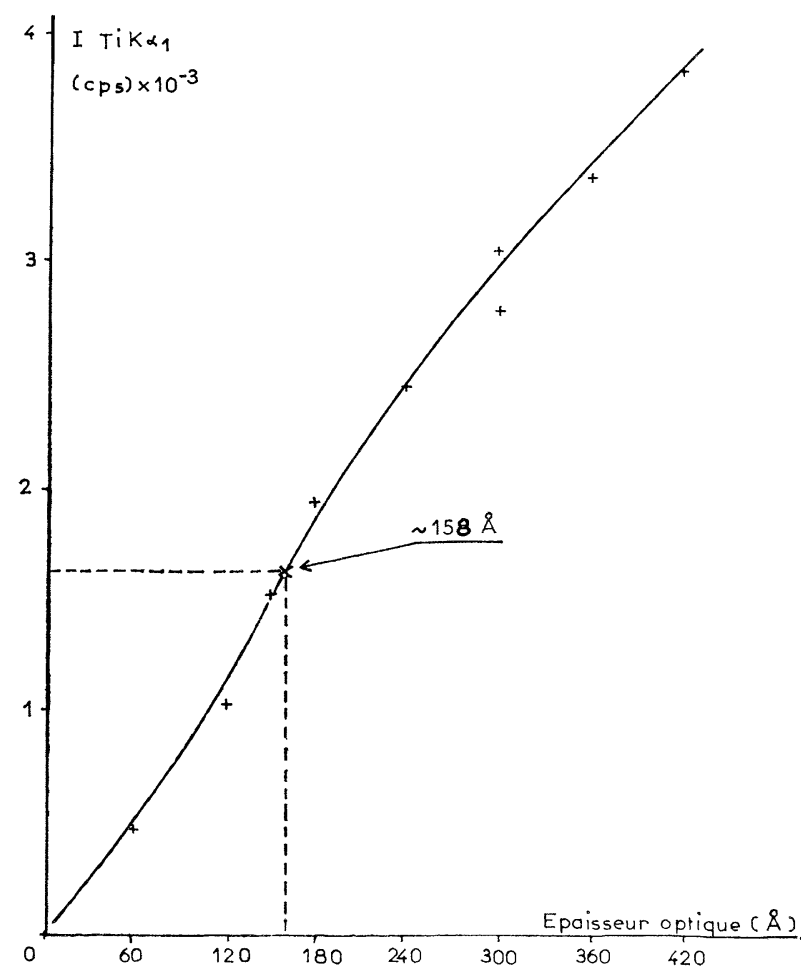

Frg. 5. $-I(\mathrm{Ti} K \alpha)=f$ (épaissur optique). Couchede $\mathrm{TiO}_{2}$, substrat $\mathrm{SiO}_{2}$. Conditions: $50 \mathrm{kV}, 20 \mathrm{~mA}$.

Les variations de $b$ résultent des variations relatives $\Delta c / c$ pour la composition chimique, $\Delta \mu / \mu$ pour les coefficients d'absorption et $\Delta \rho / \rho$ pour la densité.

Or ce coefficient présente, sur des supports en silice amorphe, un maximum pour une épaisseur de couche mince de titane de $\simeq 307 \AA$ et de $\simeq 158 \AA$ pour l'oxyde de titane (fig. $7-8$ ). Nous pensons que ces anomalies du facteur $b$ traduisent une évolution dans le processus de croissance des couches par l'intermédiaire de $\Delta \rho / \rho$ que l'on pourrait comparer au coefficient de remplissage.

La fluorescence de rayons $\mathrm{X}$ permet donc, comme nous venons de le voir, de préciser le processus de 


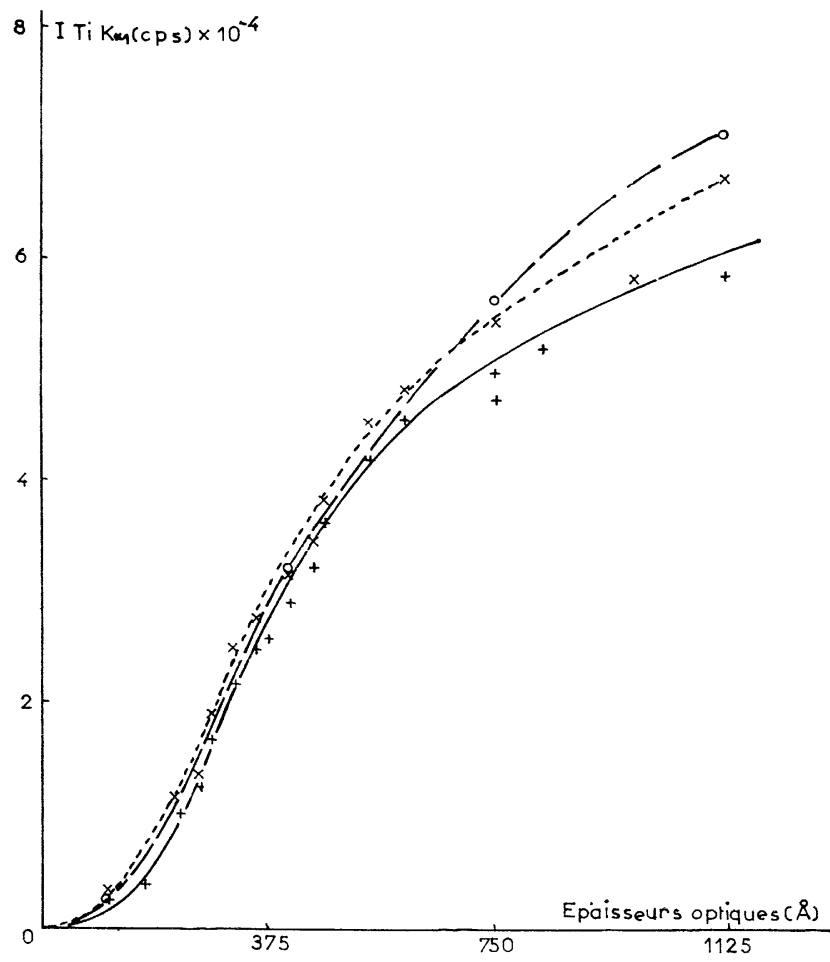

FIG. 6. $-I\left(\mathrm{Ti} K \alpha_{1}\right)=f($ épaisseur optique $)$ Couche de Ti. Substrat :,$+ \mathrm{SiO}_{2} ; \times$, verre ; $\bigcirc$, aluminium.

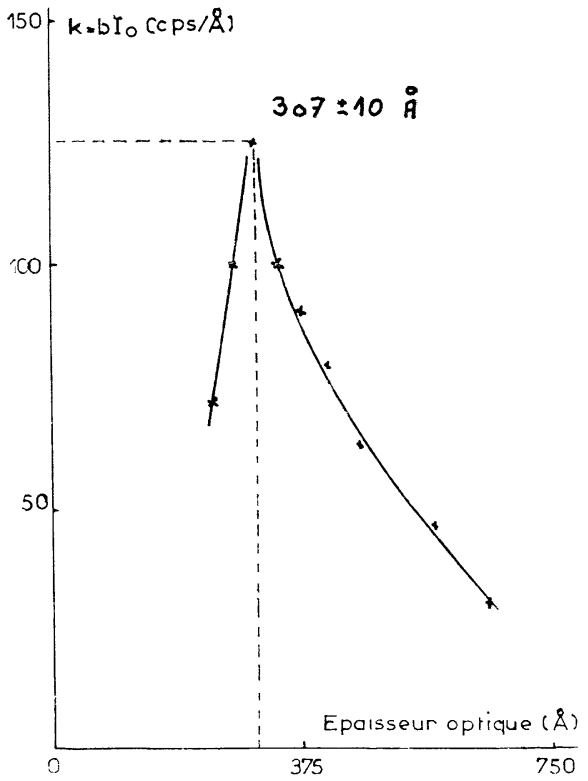

FIG. 7. $-b=k / I_{0}$; couche $\mathrm{Ti}$, substrat $\mathrm{SiO}_{2}$. Conditions $50 \mathrm{kV}, 20 \mathrm{~mA}$.

germination caractéristique du couple supportcouche mince. En effet, en modifiant la nature du substrat, on modifie le nombre et l'énergie des sites de germination qui influencent non seulement la

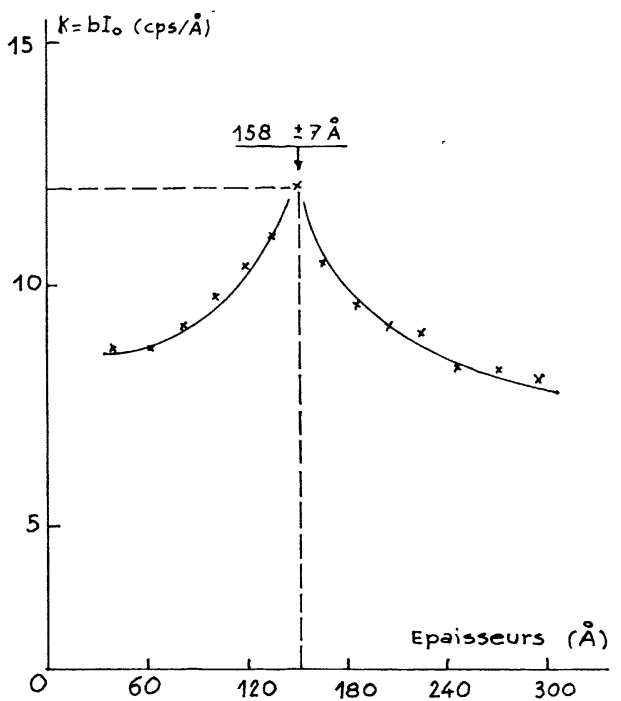

FIg. 8. - Couche $\mathrm{TiO}_{2}$, substrat $\mathrm{SiO}_{2}$. Conditions $50 \mathrm{kV}, 20 \mathrm{~mA}$.

structure de la couche, mais aussi son épaisseur et la nature chimique de la couche de transition.

Les propriétés physiques, chimiques et cristallographiques de la couche mince proprement dite dépendent enfin fortement des caractéristiques du plasma initiateur.

Dans le cas de la condensation d'un plasma $(\mathrm{Cu}, \mathrm{A}, \mathrm{O})^{*}$, le fait que l'on puisse obtenir des couches métalliques pures par une décharge dans l'oxygène pur nous a conduit à admettre que les réactions chimiques aboutissant à la synthèse d'oxydes sont initiées à l'échelle moléculaire au sein du plasma et à les rechercher.

Nous nous baserons sur le fait que l'on observe une grande abondance relative d'atomes de $\mathrm{Cu}$ II, $\mathrm{O}$ I dans un plasma $(\mathrm{Cu}, \mathrm{A}, \mathrm{O})^{*}$ soumis à un champ électrique $\mathbf{E}$ faible et générateur de $\mathrm{Cu}_{2} \mathrm{O}$.

Dans un plasma soumis à un champ $\mathbf{E}$ moyen générateur de $\mathrm{CuO}$ on observe une abondance privilégiée de $\mathrm{Cu}$ II et $\mathrm{O}$ II. Enfin dans le cas d'un plasma soumis à un champ $\mathbf{E}$ élevé et générateur de $\mathrm{Cu}$, on constate une augmentation des $\mathrm{Cu} \mathrm{I}$ tout à fait anormale [8] (fig. 9).

Il est très probable que des niveaux métastables interviennent dans les processus chimiques de la condensation.

Nous avons tenté dès lors de formuler une théorie phénoménologique de la pulvérisation cathodique réactive [9] en nous basant sur l'hypothèse d'une succession des mécanismes élémentaires suivants :

1) Impact d'ions sur la cathode et éjection d'atomes métalliques.

2) Excitation de ces atomes au sein du plasma.

3) Condensation de ce plasma sur le substrat sous forme de couches minces. 


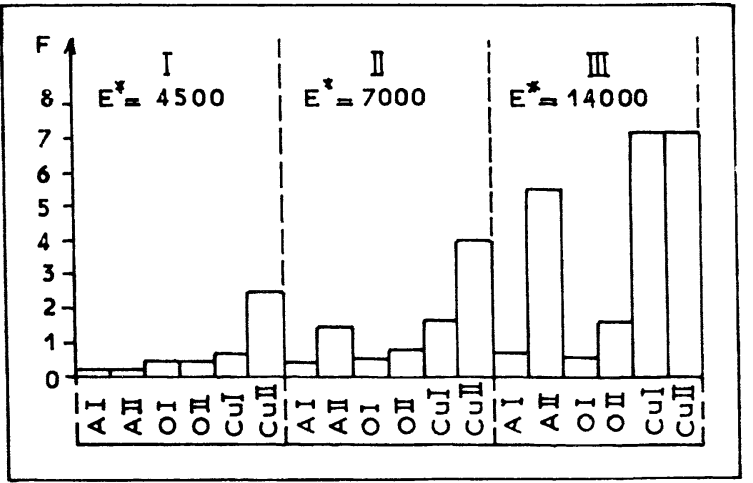

FIG. 9.

1. Impact d'ions sur la cathode et éjection d'atomes métalliques. - Le calcul est basé sur la théorie de Keywell [10]. Cependant celle-ci nécessite la connaissance du nombre d'ions projectiles frappant la cathode par unités de temps et de surface ainsi que leurs énergies. Ces deux grandeurs ne nous étant pas accessibles directement par l'expérience nous avons dû les évaluer autrement.

a) Détermination DU Nombre $N_{\mathrm{j}}$ D'Ions PRojecTIles. - Dans un même champ de force le nombre de particules déplacées est proportionnel aux coefficients de diffusion. Donc si nous faisons l'hypothèse que la viscosité du plasma est identique pour les ćlectrons et les ions nous pouvons écrire :

$$
\frac{N_{\mathrm{e}}}{N_{\mathrm{i}}}=\frac{\mathscr{D}_{\mathrm{e}}}{\mathcal{D}_{\mathrm{i}}}=\sqrt{\frac{m_{\mathrm{i}}}{m_{\mathrm{e}}}}
$$

$\left(N_{\mathrm{e}}=\right.$ nombre d'électrons par $\mathrm{m}^{2}$ par seconde; $N_{\mathrm{i}}=$ nombre d'ions par $\mathrm{m}^{2}$ par seconde; $m_{\mathrm{e}}=$ masse de l'électron ; $m_{\mathrm{i}}=$ masse de l'ion ; $\mathfrak{D}_{\mathrm{e}}=$ coefficient de diffusion des électrons; $\mathfrak{D}_{\mathrm{i}}=$ coefficient de diffusion des ions).

Nous admettrons par ailleurs que le courant total entre l'anode et la cathode est uniquement dû à un transport d'électrons. Par conséquent nous pouvons déterminer $N_{\mathrm{e}}$ à partir des caractéristiques électriques macroscopiques du plasma. D'où

et d'après (I)

$$
N_{\mathrm{e}}=E^{*} d p(R e)^{-1}
$$

$$
N_{\mathrm{i}}=\frac{E^{*} d p}{R e}\left(\frac{m_{\mathrm{e}}}{m_{\mathrm{i}}}\right)^{1 / 2}
$$

( $R=$ résistance apparente du plasma ; $e=$ charge de l'électron ; $\boldsymbol{d}=$ distance de l'anode à la cathode).

b) Détermination de L'́́nergie moYenne $W_{i}^{\circ}$ DES IONS PROJECTILES incidents. - Les $N_{\mathrm{i}}$ ions créés entreront en collision dans le plasma. A une distance $\lambda_{\Delta O}$ de la cathode, la somme des vitesses est statistiquement nulle, l'ensemble des ions n'étant alors animé que de sa vitesse de diffusion. Leur énergie moyenne au moment de l'impact sur la cathode sera alors égale à :

$$
W_{\mathrm{i}}^{\mathrm{o}}=E^{*} \text { pe } \lambda_{\mathrm{Ao}} .
$$

Portons ces valeurs dans la formule de Keywell. Nous obtenons ainsi le nombre $n_{M e}$ de particules métalliques éjectées de la cathode (spectroscopiquement pure) par unité de temps et de surface. D'où

$$
\begin{aligned}
& n_{M \mathrm{e}}=\frac{d}{R}\left[\frac{m_{\mathrm{e}} \varepsilon \lambda_{\mathrm{AO}}}{m_{\mathrm{i}} e W_{\mathrm{d}}}\right]^{1 / 2} p^{3 / 2} E^{* 3 / 2} \\
& \sum_{\mu=1}^{\mu=\mu_{\max }}\left(f_{\mathrm{p}}+1,32 \delta \mu_{1} f_{\mathrm{r}}\right) \exp -\alpha \sqrt{\mu} \cdot \exp -\frac{\xi}{2}(\mu-1)
\end{aligned}
$$

$W_{\mathrm{d}}$ représente l'énergie de déplacement, c'est-à-dire la valeur minimum de $W_{i}^{\text {o }}$ conduisant à l'éjection d'un atome métallique. En effet un ion projectile après $u$ chocs efficaces successifs avec la cathode voit son énergie décroître jusqu'ì $W_{\mathrm{d}} ; \varepsilon$ est la probabilité de choc efficace entre un ion incident et les at:mes de masse $M$ constitutifs de ia cathode ;

avec

$$
\mu_{\max }=\frac{1}{\xi} \operatorname{Ln} \varepsilon \frac{W_{\mathrm{o}}^{\mathbf{i}}}{W_{\mathrm{d}}}
$$

$$
\xi=1-\frac{1}{\varepsilon} \operatorname{Ln}\left|\frac{m_{\mathrm{i}}+M}{m_{\mathrm{i}}-M}\right| ;
$$

( $f_{\mathrm{p}}$ représente la proportions d'ions incidents pénétrant dans la cathode, proportion directement responsable de l'émission d'atomes métalliques; $f_{\mathbf{r}}$ représente la proportion d'ions incidents qui conduisent à un déplacement superficiel des atomes métalliques, déplacement qui modifie leur énergie potentielle; $\delta_{\mu 1}$ est tel que $\delta_{\mu 1}=1$ si $\mu=1$ et $\delta_{\mu 1}=0$ si $\mu \neq 1 ; \alpha$ coefficient de proportionnalité).

Dans notre cas la somme $\sum$ de la formule (III) varie peu avec $\mu$ donc avec $E^{*}$ et nous posons $\Sigma \cong$ constante.

2. Excitations dans le plasma. - Soit $n_{\text {Me }}^{*}$ le nombre de particules, fraction de $n_{\mathrm{Me}}$, qui, à la suite d'un transfert d'énergie $W_{1}$ ont vu leur structure électronique modifiée et constituent ainsi de véritables germes pour la réaction d'activation $G$

$$
n_{\mathrm{Me}}^{*}=n_{\mathbf{M e}}\left[\exp \left(-\frac{G}{W_{1}}\right)\right] \bar{f}_{\mathrm{s}} \bar{f}_{\pi}
$$

Les facteurs $\bar{f}_{\mathrm{s}}$ et $\overline{f_{\pi}}$ dépendent de la répartition des énergies de chocs selon les angles d'approche et les masses respectives des projectiles d'une part, et de leurs états électroniques d'autre part. Cependant, dans des chocs ultérieurs, une fraction de germes $n_{\text {Me }}^{*}$ sera désactivée. Désignons par $N_{1}^{*}$ le 
nombre de germes métalliques qui conduiront effectivement à la synthèse considérée :

$$
N_{1}^{*}=n_{\mathrm{Me}}^{*} \mathfrak{J}
$$

où $\mathfrak{J}$ représente la probabilité de non-désactivation d'un germe $n_{\text {Me }}^{*}$.

Si nous considérons les germes comme indépendants les uns des autres, leur répartition de vitesse obéit à la statistique de Maxwell. D'où

$$
J=\int_{\theta}^{\infty}\left(\frac{t}{\tau}\right)^{2} \exp -\left(\frac{t}{\tau}\right)^{2} \mathrm{~d} t
$$

où $t=$ temps de vol de la particule; $\tau=$ ibre temps moyen; $\theta=$ temps de relaxation de la réaction d'énergie d'activation $G$.

Les développements de cette intégrale sont de la forme

$$
\mathfrak{J}=\left(\frac{\theta}{\tau}\right)^{\beta} \exp \left[-\left(\frac{\theta}{\tau}\right)^{2}\right] \frac{2}{\sqrt{ } \pi} \mathcal{R}
$$

où $\mathcal{R}$ représente alors une série convergente constante. Dans notre cas $\beta$ est voisin de l'unité.

Par ailleurs $\tau=\lambda_{\mathrm{Me}} / \bar{u}, \bar{u}$ étant la vitesse quadratique moyenne des particules métalliques dont le libre parcours moyen est $\lambda_{M \mathrm{e}}$.

$$
\text { Or } \bar{u}=\left[\frac{2 \bar{W}}{M}\right]^{1 / 2} \text { et un raisonnement analogue à }
$$

celui que nous avions effectué lors du calcul de l'énergie $W_{\mathbf{i}}^{\mathbf{o}}$ nous permet d'écrire que

$$
\tau=\lambda_{M \mathrm{e}}\left[\frac{M}{2 p \lambda_{\mathrm{AO}} e}\right]^{1 / 2}\left(E^{*}\right)^{-1 / 2} .
$$
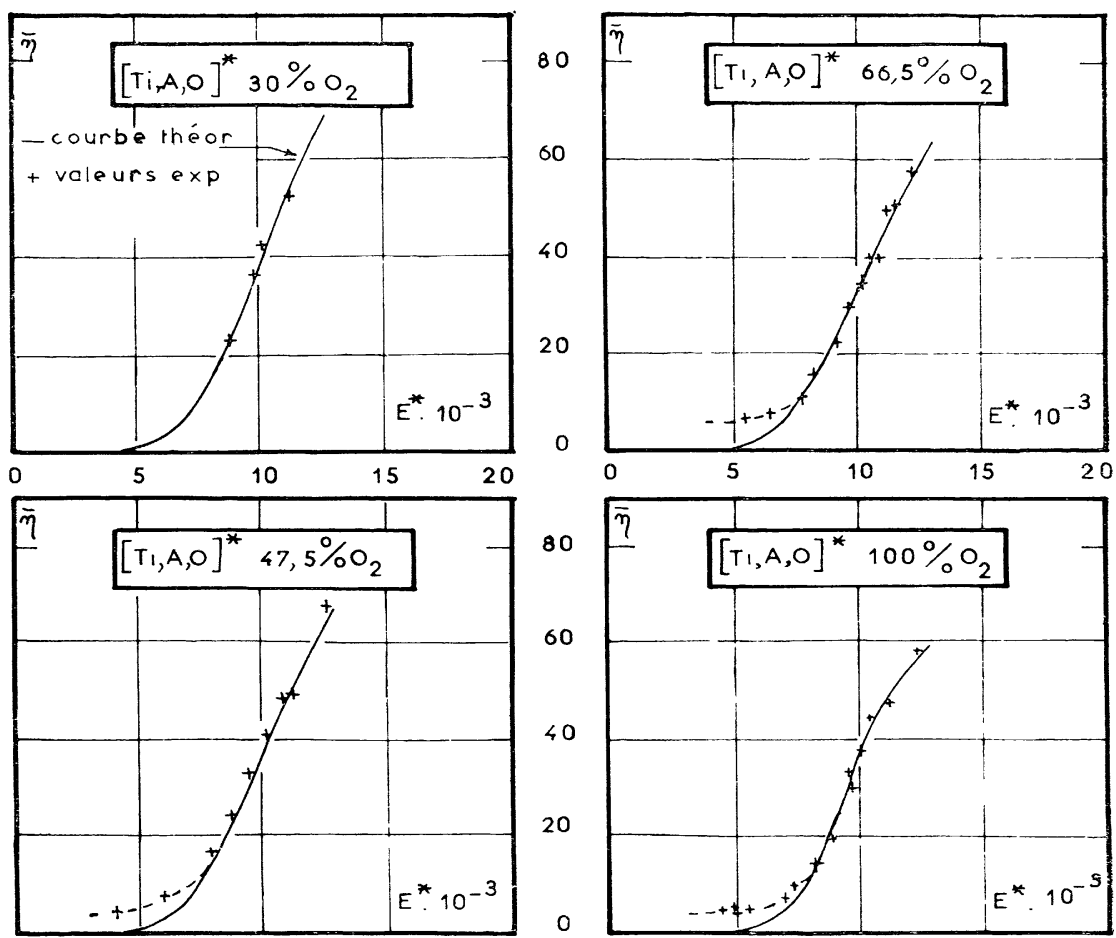

FIG. 10.
Calculons maintenant l'énergie transférée $W_{1}$, introduite en (IV).

Nous pouvons considérer, dans la zone de réaction, le plasma comme sensiblement isotrope. Le champ électrique faible intervient alors comme une faible perturbation et par conséquent la vitesse est une anisotropie du premier ordre, donc l'énergie d'échange $W_{1}$ est proportionnelle à $E^{*}[11]$

$$
W_{1}=P E^{*}
$$

$P=$ facteur de proportionnalité.

Compte tenu de l'ensemble de ces considérations l'équation (V) devient :

$$
\begin{gathered}
N_{1}^{*}=\frac{\mathrm{p}^{2} \mathrm{~d} \theta}{R} \lambda \bar{M}_{\mathrm{e}}^{1} \lambda_{\mathrm{AO}} \bar{f}_{\mathrm{s}} \bar{f}_{\pi} S \Re \frac{2}{\sqrt{ } \pi}\left(\frac{2 m_{\mathrm{e}} \varepsilon}{m_{\mathrm{i}} W_{\mathrm{d}} M}\right)^{\mathbf{1 / 2}} \\
E^{* 2} \exp -\left(\frac{G}{P E^{*}}+\frac{2 \theta^{2} p \lambda_{\mathrm{AO}} \lambda_{M \mathrm{e}} e E^{*}}{M}\right)
\end{gathered}
$$

équation de la forme

$$
N_{1}^{*}=A^{\prime} E^{* 2} \exp -\left(\frac{B}{E^{*}}+C E^{*}\right) .
$$

3. Condensation sur support amorphe. - La quantité $N_{1}^{*}$ représente alors le flux de germes métalliques par seconde aboutissant à la synthèse du composé chimique condensé préférentiellement dans la couche mince. Nous faisons l'hypothèse que ce nombre est directement lié au rendement $\bar{\eta}$ 
(en $\AA / m n$ ) de la condensation qui est la grandeur accessible à l'expérience. D'où

$$
\bar{\eta}=A E^{* 2} \exp -\left(\frac{B}{E^{*}}+C E^{*}\right) .
$$

Nous avons appliqué cette dernière relation à la condensation de plasmas ( $\mathrm{Ti}, \mathrm{A}, \mathrm{O}) *($ fig. 10). Nous constatons le bon accord existant entre les valeurs théoriques et expérimentales pour les valeurs élevées de $E^{*}$. Toutefois pour les faibles valeurs du champ réduit le rendement expérimental est toujours plus élevé que le rendement théorique. Cette discordance peut trouver son origine dans les particularités des processus de germination, dans une détermination imprécise du nombre de particules éjectées de la cathode et dans les processus d'oxydation initiale. Notons par ailleurs, que de la connaissance du coefficient $C$, nous pouvons déduire la valeur du temps de relaxation $\theta$ de la réaction la plus lente du processus physico-chimique aboutisasnt à la synthèse du composé chimique condensé sous forme de couche mince. Cette durée varie alors avec la composition du mélange gazeux initial tout en restant voisine de $10^{-3}$ secondes. Le tableau suivant récapitule les valeurs numériques des coefficients $A, B, C$ et $\theta$ dans le cas de la condensation d'un plasma ( $\mathrm{Ti}, \mathrm{A}$, $\mathrm{O})^{*}$ :

\begin{tabular}{|c|c|c|c|c|}
\hline$\% \mathrm{O}_{2}$ & $A$ & $B \times 10^{-}$ & $<10^{4}$ & $\theta \times 10^{3}$ \\
\hline & 367 & 1051 & 7566 & 142 \\
\hline 21 & $6,07 \cdot 10^{-3}$ & 5,645 & 3,999 & 11,0 \\
\hline 30 & $8,21 \cdot 10^{-4}$ & 4,46 & 3,150 & 9,0 \\
\hline 47,5 & $6,62.10^{-4}$ & 4,37 & 3,092 & 7,8 \\
\hline 5 & $9,07 \cdot 10^{-4}$ & 4,79 & 3,400 & 7,0 \\
\hline 100 & 2,126 & 8,45 & 7,075 & 7,9 \\
\hline
\end{tabular}

Notons enfin que ces techniques sont également applicables à la préparation de couches minces d'halogénures, de sulfures, d'alliages, de semiconducteurs dopés, etc... Nous nous permettons de présenter à l'auditoire un échantillonnage de couches minces de $\mathrm{SiO}_{2}, \mathrm{TiO}_{2}, \mathrm{Al}_{2} \mathrm{O}_{3}, \mathrm{Cu}_{2} \mathrm{O}, \mathrm{CdS}, \mathrm{AgI}$ et $\mathrm{PbI}_{2}$, obtenues à partir de la condensation de plasmas froids sur des supports en silice, soit par évaporation réactive, soit par pulvérisation cathodique réactive.

\section{BIBLIOGRAPHIE}

[1] Perny (G.), Thèse, Strasbourg, 1957 ; Z. Ang. Chemie, $1957 ; J$. Physique, 1958, 19, 119 A.

[2] Holland, Vacuum deposition of thin films, Londres, 1958.

[3] Perny (G.) et Laville Saint Martin (B.), Colloque international sur les problèmes fondamentaux de la Physique des Couches Minces, Göttingen, septembre 1965.

[4] Perny (G.) et Laville Saint Martin (B.), C. $R$. Acad. Sc., 1965, 260, 3639 ; C. R. Acad. Sc., 1965, 260, 3949.

[5] Perny (G.), Laville Saint Martin (B.), Van den Berghe (P.) et Muller (Mlle L.), C.R. Acad. Sc., 1964, 259, 1852.
[6] Arntz (F.) et Chernow (F.), J. Vac. Sc. Tech., 1965, 2, 1, 20-23.

[7] Van den Berghe (P.) et Perny (G.), J. Physique, 1966, 27, No 11-12 (à paraître).

[8] Perny (G.) et Samirant (M.), C. R. Acad. Sc., 1965, 260, 5009.

[9] Perny (G.), Samirant (M.) et Laville Saint Martin (B.), C. R. Acad. Sc., 1966, 262, série C, 1841.

[10] Keywell (F.), Phys. Res., 1955, 97, no 6, 1611.

[11] Delcroix (J.), Introduction à la théorie des gaz ionisés, Dunod Paris. 